\title{
Performance Evaluation of SiPM Photosensors in the Presence of Magnetic Fields
}

\author{
S. España ${ }^{1}$, J. Cal-González ${ }^{1}$, L.M. Fraile ${ }^{1}$, E. Picado ${ }^{1}$, J.L. Herraiz ${ }^{1}$, E. \\ Vicente $^{1,3}$, J.M. Udías ${ }^{1}$, M. Desco ${ }^{2}$, J.J. Vaquero ${ }^{2}$ \\ ${ }^{l}$ Grupo de Física Nuclear, Dpto. Física Atómica, Molecular y Nuclear, Universidad Complutense, \\ Madrid, Spain \\ ${ }^{2}$ Unidad de Medicina y Cirugía Experimental, Hospital General Universitario Gregorio Marañón, \\ Madrid, Spain \\ ${ }^{3}$ Instituto de Estructura de la Materia, Consejo Superior de Investigaciones Científicas (CSIC), \\ Madrid, Spain
}

\begin{abstract}
The Multi Pixel Photon Counter (MPPC) or Silicon Photomultiplier (SiPM) consists of an array of Geiger-mode photodiodes (microcells). It is a promising device for PET thanks to its high photon detection efficiency (PDE) and immunity to high magnetic fields. It is also very easy to use, with simple electronic read-out, high gain and small size. In this work we evaluate the performance of three $1 \times 1 \mathrm{~mm}^{2} \mathrm{SiPM}$ and one $2 \times 2$ SiPMs array $\left(6 \times 6 \mathrm{~mm}^{2}\right.$ of active area). We examine the dependence of the energy resolution and the gain of these devices on the temperature and reverse bias when coupled to MLS and LYSO scintillator crystals. The good performance of these devices up to 7 Tesla has been also confirmed.
\end{abstract}

Keywords: Positron Emission Tomography (PET), Silicon Photomultipliers (SiPM) PACS: 29.30.Kv; 29.40.-n; 85.60.Dw

\section{INTRODUCTION, MATERIALS AND METHODS}

The recently introduced [1] Multi Pixel Photon Counters (MPPC), also know as Silicon Photomultipliers (SiPM), exhibit high photon detection efficiency (PDE), are immune to magnetic fields, easy to use with simple read out electronics and have small size. Therefore, they are ideal components for PET/MRI and In-Beam PET detectors.

Devices with 100, 400 and 1600 microcells $\left(1 \times 1 \mathrm{~mm}^{2}\right)$ were coupled to $1.5 \times 1.5 \mathrm{x}$ $12 \mathrm{~mm}^{3}$ MLS crystal. A $2 \times 2$ array, with elements of $3 \times 3 \mathrm{~mm}$ and 3600 microcells each, was coupled to a $10 \times 10 \times 20 \mathrm{~mm}^{3}$ LYSO crystal and to and array of $4 \times 4$ MLS crystals of $1.5 \times 1.5 \times 12 \mathrm{~mm}^{3}$. The devices were placed in the static magnetic field of a 7 Tesla superconducting magnet (BIOSPEC 70/20, Bruker Corporation). 


\section{RESULTS}

FWHM energy resolution of $20 \%$ at $511 \mathrm{keV}$ was attained for the best single SiPM, the 1600 microcells one. The variation of energy resolution and relative gain of individual SiPM with the temperature and applied voltage were measured [2], (figure 1). The SiPM array showed energy resolution ranging from $12 \%$ to $22 \%$ at $511 \mathrm{keV}$ and $5 \%$ to $6 \%$ at $1275 \mathrm{keV}$. All the $4 \times 4$ crystals of the crystal matrix were perfectly resolved by the $2 \times 2$ SIPM array, yielding a 10:1 peak to valley ratio (figure 2). These results were independent on the magnetic field applied.
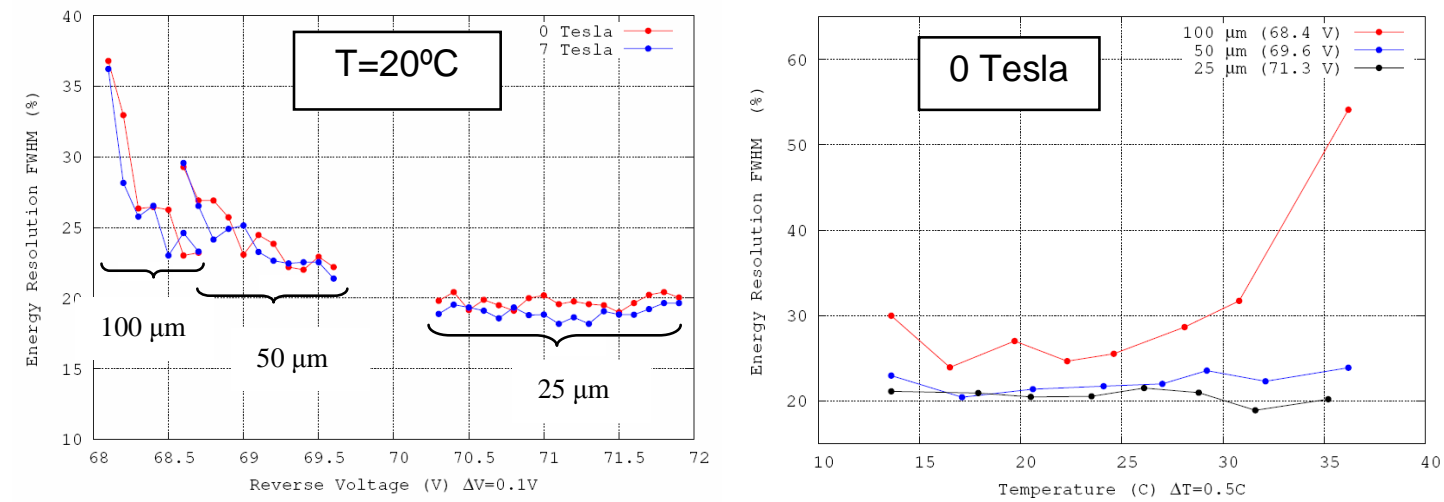

FIGURE 1. Variation of energy resolution with the reverse voltage applied at $\mathrm{T}=20^{\circ} \mathrm{C}$ (left) and with the Temperature (right), to individual SiPMs.

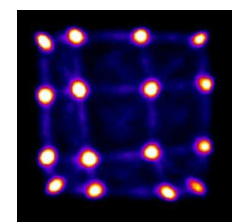

$18 \mathrm{~F}$

$68.2 \mathrm{~V}-20^{\circ} \mathrm{C}$

$5 \cdot 10^{5}$ counts

Software

Anger logic

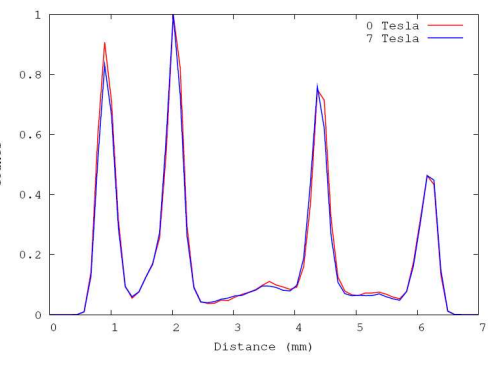

Peak to valley ratio $=10: 1$

FIGURE 2. Separation of $4 \times 4$ MLS crystal matrix with $2 \times 2$ SiPMs array.

\section{ACKNOWLEDGMENTS}

This work has been supported in part by MEC (FPA2007-62216), CDTEAM (Programa CENIT, Ministerio de Industria), UCM (Grupos UCM, 910059), CPAN (Consolider-Ingenio 2010) CSPD-2007-00042 and the RECAVA-RETIC network.

\section{REFERENCES}

1. Otte, A.N. et al., A test of Silicon Photomultipliers as readout for PET. Nucl. Inst. Meth. In Phy. Res. A, 2005. 545(3): p. 705-15..

2. S. España et al., Performance Evaluation of SiPM Detectors for PET Imaging in the Presence of Magnetic Fields, IEEE NSS-MIC, 2008. 